\title{
Modifying the Radiative Quantum Efficiency of Erbium-Doped Glass in Silicon Slot Waveguides
}

\author{
Ryan M. Briggs, Gerald M. Miller, and Harry A. Atwater \\ Thomas J. Watson Laboratory of Applied Physics \\ California Institute of Technology, Pasadena, California 91125 USA \\ Email: rbriggs@caltech.edu
}

\begin{abstract}
We have modulated the radiative emission rate of $\mathrm{Er}$ in $\mathrm{Si}$ slot waveguides with Er-doped glass slots by varying the waveguide layer dimensions. The corresponding quantum efficiency of $1537-\mathrm{nm}$ emission varies between 0.37 and 0.51 .
\end{abstract}

\section{INTRODUCTION}

High index contrast Si-based slot waveguides exhibit useful properties owing to extremely large relative electric field intensities in the slot layer for guided transverse-magnetic (TM) modes [1]. In the pursuit of an efficient light source integrated with $\mathrm{Si}$ photonic devices, slot waveguides with actively emitting slot layers are intriguing since they are expected to produce modal gain proportional to the intensity of the electric field confined to the slot, which can exceed $50 \%$ for certain geometries [2]. Another consequence of this confinement is the modification of the local density of optical states (LDOS), which also scales with the electric field intensity but depends on all modes, both guided and radiative, supported by the waveguide structure [3], [4]. This LDOS enhancement results in concomitant enhancement of the radiative component of the spontaneous emission rate and increased coupling efficiency into TM modes, as shown in recent work on Si slot waveguides with Er-doped silica [5] and Si nanocrystal sensitized Er slot layers [6].

Er-doped glass is an attractive candidate as an active slot material since the ${ }^{4} I_{13 / 2}$ to ${ }^{4} I_{15 / 2}$ transition of $\mathrm{Er}^{3+}$ produces light near $1550 \mathrm{~nm}$. In addition to being in the telecommunications $\mathrm{C}$ band, light near this wavelength is below the $\mathrm{Si}$ band edge and can thus propagate through $\mathrm{Si}$ with low loss. Furthermore, the low index of silica-based glass is ideal for achieving large electric field confinement in slot waveguides.

We have fabricated planar Si slot waveguides with active Erdoped soda-lime glass slot layers and varied Si layer dimensions in order to demonstrate the dependence of the radiative spontaneous emission rate on guided waveguide modes. Timeresolved photoluminescence (PL) from the waveguides was used to determine the total emission rate of $\mathrm{Er}^{3+}$ ions in the glass slot layers. For each slot structure, the interfaces of the Er-doped layer, as well as the constituents of layer itself, are identical; therefore, the non-radiative decay rate, $\Gamma_{\mathrm{nr}}$, can be assumed constant and all observed changes in the total emission rate can be attributed to changes in the LDOS.

Calculations of the LDOS for the slot structures are presented and compared with measured values of the radiative emission rate using $\Gamma_{n r}$ as a sole fitting parameter. The calculations were performed using an extension of the formalism laid out by Urbach and Rikken for dielectric slabs [7]. We find that the measured values of the emission rate match well to the theoretical predictions. Knowing $\Gamma_{\mathrm{nr}}$ and the LDOS allows the internal quantum efficiency, $\eta$, to be determined. We find that $\eta$ ranges from 0.37 to 0.51 for the waveguide geometries considered here, corresponding to a significant change that is a function of waveguide geometry alone.

\section{FABRICATION}

Planar slot waveguide structures were fabricated by first thinning the top Si layer of Si-on-insulator (SOI) samples using thermal oxidation and etching with buffered hydrofluoric acid. The SOI had a lightly B-doped $\left(\sim 10^{15} \mathrm{~cm}^{-3}\right)$, ptype $\mathrm{Si}$ device layer and a $3-\mu \mathrm{m}$ buried oxide layer. Samples were prepared with device layer thicknesses of approximately 100, 150, and $200 \mathrm{~nm}$. An additional 5-nm thermal oxide passivation layer was grown on the top surfaces of the samples. A 45-nm layer of Er-doped soda-lime glass was then deposited on all the samples simultaneously using DC ion beam cosputtering of $\mathrm{SiO}_{2}$ with soda-lime glass and metallic Er. The samples were annealed for $300 \mathrm{~s}$ in a rapid thermal annealing (RTA) furnace at $850^{\circ} \mathrm{C}$ in an $\mathrm{O}_{2}$ ambient. The RTA furnace activates $\mathrm{Er}^{3+}$ ions in the glass matrix with minimal oxide undergrowth $(\sim 1 \mathrm{~nm})$ beneath the film. Spectroscopic ellipsometry was used to verify that the total thickness of oxide on the SOI was approximately $51 \mathrm{~nm}$ for all samples, and Rutherford backscattering spectroscopy (RBS) was used to verify that Er resides only in the top $45 \mathrm{~nm}$. RBS also revealed that the films contain 1.1 at. \% Er.

An amorphous Si layer was sputtered atop the Er-doped layers and annealed in the RTA furnace for $300 \mathrm{~s}$ at $700{ }^{\circ} \mathrm{C}$ in $\mathrm{N}_{2}$ to crystallize the film. Ellipsometry revealed polycrystalline optical properties over the visible to near-infrared spectrum and a film thickness of $73 \mathrm{~nm}$. The final planar waveguide geometry is shown in Fig. 1. As described in Section IV, the radiative emission rate in bulk glass, $\Gamma_{\text {rad }}^{1.45}$, was determined by sputtering an identical Er-doped film on a silica substrate.

\section{LDOS CALCULATIONS}

For an electron bound to an $\mathrm{Er}^{3+}$ ion, Fermi's golden rule dictates that the transition rate from an excited state to the ground state via photon emission is proportional to the density 


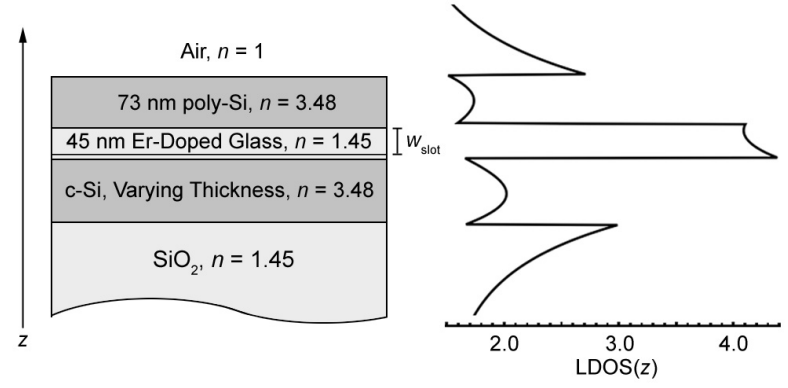

Fig. 1. Planar slot waveguide geometry shown with the calculated LDOS corresponding to a crystalline Si layer thickness of $100 \mathrm{~nm}$.

of optical states. In contrast to emitters in a homogeneous medium, the spontaneous emission rate of $\mathrm{Er}^{3+}$ ions in a waveguide is position dependent since the fields associated with photon states are themselves a function of position. This local density of optical states, or LDOS, is defined as the position-dependent factor that scales the radiative emission rate. In this work, the LDOS is normalized to the density of states in a homogeneous medium of $n=1.45$, so the total decay rate is

$$
\Gamma_{\text {tot }}(\mathbf{r})=\Gamma_{\text {rad }}(\mathbf{r})+\Gamma_{\mathrm{nr}}=\operatorname{LDOS}(\mathbf{r}) \Gamma_{\mathrm{rad}}^{1.45}+\Gamma_{\mathrm{nr}},
$$

where the non-radiative decay rate, $\Gamma_{\mathrm{nr}}$, accounts for effects such as Auger energy transfer and defect quenching, and $\Gamma_{\mathrm{rad}}^{1.45}$ is the radiative emission rate in bulk glass.

Taking into account all discrete guided modes and the continuum of radiative modes associated with the waveguide structure, the LDOS is calculated as in Refs. [4] and [7] for an isotropic ensemble of emitters, and, due to the symmetry, depends only on $z$ (as defined in Fig. 1). As seen in Fig. 1, the LDOS varies over the thickness of the slot. The collective effect on the radiative emission rate is captured by the averaged LDOS, defined as

$$
\operatorname{LDOS}_{\mathrm{av}}=\frac{1}{w_{\text {slot }}} \int_{\text {slot }} \operatorname{LDOS}(z) d z,
$$

where the slot width is $w_{\text {slot }}=45 \mathrm{~nm}$.

The averaged LDOS for the slot region is plotted in Fig. 2 for different thicknesses of the crystalline Si layer beneath the Er-doped film, where the top Si layer thickness is held constant at $73 \mathrm{~nm}$, and the slot layer is 51-nm thick with the top 45 $\mathrm{nm}$ containing Er emitters. As the c-Si layer becomes thicker, the waveguide begins to support more guided modes. As each guided mode is born, there is a slope discontinuity in the curve representing the contribution from radiative modes. The net effect is that $\mathrm{LDOS}_{\mathrm{av}}$ for all modes is a smoothly varying function. Due to large electric field confinement in the slot, the contribution from the first-order TM mode becomes quite large as the c-Si layer approaches a thickness of around 200 $\mathrm{nm}$. Overall, the averaged LDOS is modulated by a factor of 3 simply by adjusting the thickness of the c-Si layer. Consequently, one can expect large changes in the observed emission rate with changes in geometry.

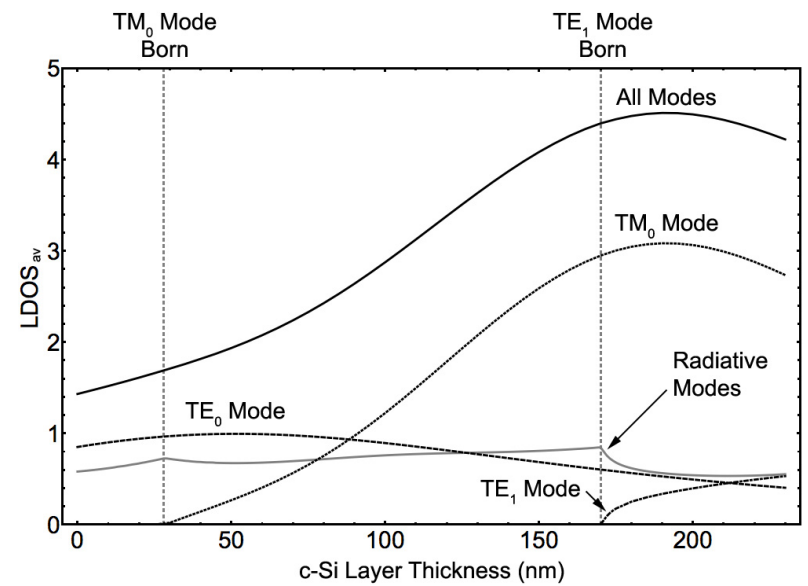

Fig. 2. The calculated averaged LDOS for the slot region of the Si slot waveguide geometry as a function of the thicknesses of the crystalline $\mathrm{Si}$ layer beneath the Er-doped film. The top Si layer thickness as well as the thickness of the slot are constant. Contributions from each guided mode, both transverse electric (TE) and transverse magnetic (TM), are shown, as is the contribution due to all radiative modes.

\section{Time-Resolved PL Measurements}

Emission lifetimes for the Er-doped structures were obtained using the 488-nm line of an Ar gas laser passed through an acousto-optic modulator. Er-related emission near $1550 \mathrm{~nm}$ was collected through a monochromator and into a liquid $\mathrm{N}_{2}$ cooled infrared photomultiplier. For the data presented here, the PL intensity from each sample scaled linearly with pump power, indicating the absence of saturation effects. A representative PL spectrum is shown in the inset of Fig. 3. Upon blocking the pump laser in each modulation period, the time-resolved PL intensity at $1537 \mathrm{~nm}$ was recorded with a multichannel scaler.

\section{A. Characterization of Er-Doped Glass Films}

For reference, we measured the radiative emission rate of the Er-doped films corresponding to a homogeneous medium with $n=1.45$. Note that this rate, $\Gamma_{\mathrm{rad}}^{1.45}$, is the radiative rate when $\operatorname{LDOS}=1$. For a $45-\mathrm{nm}$ Er-doped glass film on $\mathrm{SiO}_{2}$, the PL lifetime, $\tau$, was $11.44 \pm 0.01 \mathrm{~ms}$. By placing oil with an index of 1.518 on the surface of the sample, which is assumed to not change the non-radiative decay rate, the calculated LDOS is increased from 0.764 to 1.047. Indeed, $\tau$ is decreased to $9.45 \pm 0.01 \mathrm{~ms}$ with the fluid cover. The original lifetime was recovered once the oil was removed with isopropanol.

By solving Eq. 1 for the geometries with and without the fluid, where $\Gamma_{\text {tot }}=\tau^{-1}$, we obtain $\Gamma_{\text {rad }}^{1.45}=65 \mathrm{~s}^{-1}$, similar to previously reported values for Er in soda-lime glass [8], and $\Gamma_{\mathrm{nr}}=38 \mathrm{~s}^{-1}$. This corresponds to a bulk-glass radiative quantum efficiency of

$$
\eta=\frac{\Gamma_{\mathrm{rad}}^{1.45}}{\Gamma_{\mathrm{rad}}^{1.45}+\Gamma_{\mathrm{nr}}}=0.63 .
$$




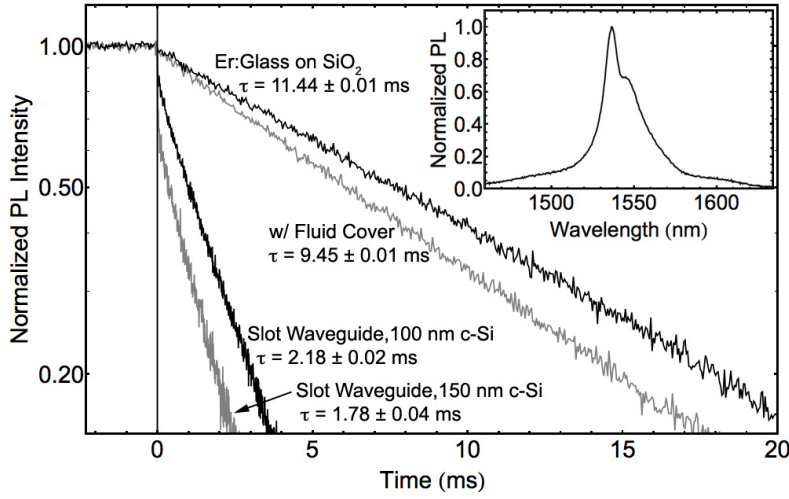

Fig. 3. Time-resolved PL for Er-doped glass films on $\mathrm{SiO}_{2}$ and in slot waveguide structures. The data for the structure with the $200-\mathrm{nm}$ c-Si layer is omitted since it overlaps the data corresponding to the $150-\mathrm{nm}$ c-Si layer. The 488-nm pump was blocked at the time marked 0, allowing the spontaneous emission lifetime, $\tau$, to be measured. For the film on $\mathrm{SiO}_{2}$, a fluid cover with $n=1.518$ was placed on the surface, resulting in an increase of the decay rate. The inset shows the Er PL spectrum, which peaks at $1537 \mathrm{~nm}$.

\section{B. Emission from Planar Si Slot Waveguides}

The PL lifetime was measured for three slot waveguide geometries with c-Si layer thicknesses around 100, 150, and $200 \mathrm{~nm}$, and the corresponding decay rates are plotted in Fig. 4. The PL intensity was low for these samples due to interference and absorption of the pump in the top Si layer, but also due to increased coupling of spontaneous emission into guided waveguide modes [4]. This effect becomes more pronounced as the $\mathrm{c}$-Si layer becomes thicker and the relative LDOS contribution from guided modes becomes larger.

The non-radiative decay rate, $\Gamma_{\mathrm{nr}}$, was adjusted to provide the best fit to the data, and was thus determined to be 288 $\mathrm{s}^{-1}$. This significant increase compared with Er-doped glass on $\mathrm{SiO}_{2}$ is due to interaction with defects at the $\mathrm{Si} / \mathrm{SiO}_{2}$ interfaces as well as in the bulk Si layers.

Overall, the differences in $\Gamma_{\text {tot }}$ for the Er-doped layers in the different waveguide geometries are well accounted for by the LDOS alone, suggesting that it is reasonable to assume a constant value of $\Gamma_{\mathrm{nr}}$. Furthermore, since $\Gamma_{\mathrm{nr}}$ is the sole fitting parameter and affects only the offset of the calculated curve in Fig. 4, these measurements serve as a tenable verification of the LDOS theory.

The observed changes in $\Gamma_{\text {rad }}$ result in modification of the internal quantum efficiency, as shown on the right axis of Fig. 4. In particular, $\eta$ increases from 0.37 for a slot waveguide with a $100-n m$ c-Si layer to 0.51 for a $200-n m$ c-Si layer. This change is purely a function of geometry, since the local environment of the $\mathrm{Er}^{3+}$ ions remains unchanged. Finally, we point out that, despite the significant increase in $\Gamma_{\mathrm{nr}}$ due to the Si layers of the slot waveguide, much of the quantum efficiency can be recovered as a result of the LDOS effect.

\section{CONCLUSiON}

We have demonstrated modification of the radiative spontaneous emission rate, and consequently the radiative quantum

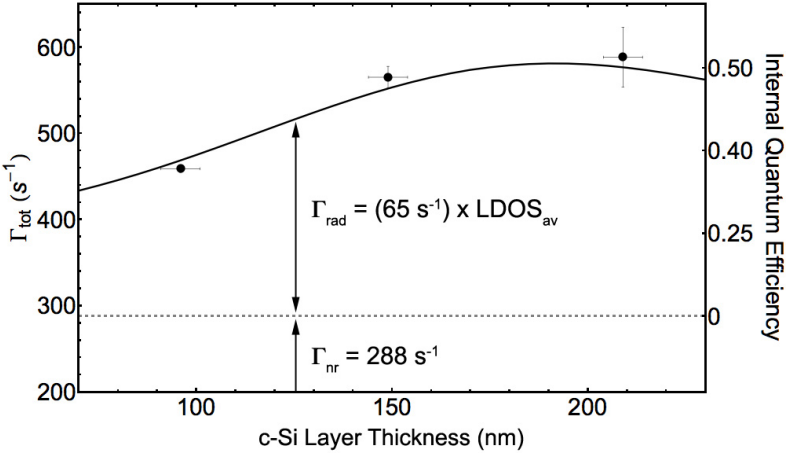

Fig. 4. Spontaneous emission rate for slot waveguides with varying thickness of the lower c-Si layer. The solid curve corresponds to the total averaged LDOS shown in Fig. 2 with the offset, $\Gamma_{n r}$, that results in the best fit to the data for fabricated structures. The corresponding radiative quantum efficiency is shown on the right axis.

efficiency, of Er-doped soda-lime glass layers in planar $\mathrm{Si}$ slot waveguides by adjusting the waveguide geometry. The films were shown to have a bulk glass radiative emission rate of $65 \mathrm{~s}^{-1}$ and a quantum efficiency of 0.63 when deposited on $\mathrm{SiO}_{2}$. Once incorporated into $\mathrm{Si}$ slot structures, the nonradiative decay rate is increased more than 7-fold; however, the radiative rate for $1537-\mathrm{nm}$ radiation was greatly enhanced due to the contribution of guided modes to the local density of optical states. Furthermore, this radiative effect was shown to vary significantly due to changes in the waveguide geometry in agreement with theoretical calculations of the local density of optical states.

Overall, this work demonstrates that one can take advantage of the large slot confinement in high index contrast slot waveguides to significantly modify the radiative properties of emitters in the slot. In particular, by carefully optimizing the waveguide geometry, the radiative quantum efficiency of Erdoped glass can be maximized, which may prove useful in realizing active Si-based waveguides operating near $1550 \mathrm{~nm}$.

\section{ACKNOWLEDGMENTS}

The authors thank D. Pacifici for useful discussions. RMB gratefully acknowledges the support of the NDSEG Fellowship. This project was funded by the AFOSR under the MURI Award FA9550-06-1-0470.

\section{REFERENCES}

[1] V.R. Almeida, Q. Xu, C.A. Barrios, and M. Lipson, Opt. Lett. 29, 1209 (2004).

[2] J.T. Robinson, K. Preston, O. Painter, and M. Lipson, Opt. Exp. 16, 16659 (2008).

[3] C. Creatore and L.C. Andeani, Phys. Rev. A 78, 063825 (2008).

[4] Y.C. Jun, R.M. Briggs, H.A. Atwater, and M.L. Brongersma, Opt. Exp.. 17, 7479 (2009)

[5] C. Creatore and L.C. Andeani, M. Miritello, R. Lo Savio, and F. Priolo, App. Phys. Lett. 94, 103112 (2009).

[6] M. Galli, D. Gerace, A. Politi, M. Liscidini, M. Patrini, L.C. Andreani, A. Canino, M. Miritello, R. Lo Savio, A. Irrera, and F. Priolo, App. Phys. Lett. 89, 241114 (2006)

[7] H.P. Urbach and G.L.J.A. Rikken, Phys. Rev. A 57, 3913 (1998)

[8] M.J.A. de Dood, J. Knoester, A. Tip, and A. Polman, Phys. Rev. B 71, 115102 (2005). 